\title{
Rabies encephalitis presenting with new-onset refractory status epilepticus (NORSE)
}

Mauricio F. Villamar, MD; Jonathan H. Smith, MD; Dianne Wilson, MD; Vanessa D. Smith, MD

A

15-year-old girl, previously healthy with normal developmental history, had an episode of unilateral arm jerking at school followed by transient loss of consciousness. Two weeks later, she presented to the emergency department with recurrent generalized convulsions despite treatment with lorazepam and fosphenytoin. In retrospect, her family reported a 1-month history of personality changes (i.e., listlessness) and decline in academic performance. There was no family history of epilepsy, metabolic disorders, or immunodeficiency. The patient resided in rural northeastern Kentucky. There was no recent travel history.

On presentation, she was afebrile, bradycardic, and minimally arousable. She had extensor posturing of the upper extremities, and withdrew both lower extremities to pain. She was intubated for airway protection and started on a propofol infusion after continuous video EEG showed generalized nonconvulsive status epilepticus. Phenytoin and phenobarbital were also used for treatment of seizures. Urine drug screen was negative and CT head was normal. She was started on empiric IV antibacterial and antiviral therapy.

Extensive diagnostic studies were performed in peripheral blood and in CSF and were all negative (table). CSF, obtained 6 hours after admission, showed 5 erythrocytes/ $\mu \mathrm{L}, 8$ leukocytes/ $\mu \mathrm{L}$ (6 lymphocytes, 2 neutrophils), elevated glucose $(98 \mathrm{mg} / \mathrm{dL})$, and elevated protein $(91 \mathrm{mg} / \mathrm{dL})$. MRI head was obtained 24 hours after admission. No abnormalities were noted on T1 (axial and coronal, both pregadolinium and postgadolinium), T2, or T2 fluidattenuated inversion recovery (axial and coronal) or diffusion-weighted imaging.

On hospital day 2, the patient developed rhabdomyolysis, severe lactic acidosis, and hypophosphatemia. These rapidly worsened, and the patient died 3 days after admission. A complete autopsy was performed. Microscopic examination of formalin-fixed, paraffinembedded brain tissue demonstrated diffuse intraparenchymal inflammation, focal neuronophagia, and rare neurons containing intracytoplasmic inclusions consistent with Negri bodies in the left parahippocampal cortex (figure). In situ RT-PCR of brain tissue was positive for rabies virus $(R A B V)$ RNA (nested primers GAGTCACTCGAATATGTC, GTATTGCCTCTCTAGCGG, GACATGTCCGGAAGACTG, and CTACAATGGATGCCGAC). Immunohistochemical staining using polyclonal and monoclonal reagents was negative for $R A B V$-specific antigen, although it is recognized that sensitivity of the test can be decreased after formalin fixation. ${ }^{1}$ Other findings included diffuse acute myocarditis and myoglobin cast nephropathy. Interestingly, multiple histoplasma granulomas were observed in lung, liver, and spleen, consistent with concurrent disseminated histoplasmosis.

Departments of Neurology (MFV, JHS) and Pathology and Laboratory Medicine (DW, VDS), University of Kentucky, Lexington.

Funding information and disclosures are provided at the end of the article. Full disclosure form information provided by the authors is available with the full text of this article at Neurology.org/cp.

Correspondence to: vanessa.smith@uky.edu

\section{Practical Implications}

Rabies encephalitis should be included in the differential diagnosis of new-onset refractory status epilepticus (NORSE) despite a normal MRI and the lack of robust cellular responses in the CSF. 


\section{Table Diagnostic studies performed in peripheral blood or serum and in CSF}

\section{Peripheral blood or serum}

Blood cultures

California encephalitis antibody panel

Eastern equine encephalitis antibody panel

Fungal complement fixation

HIV ELISA

Mycoplasma pneumoniae antibody panel

Rapid plasma reagin

St. Louis encephalitis antibody panel

Western equine encephalitis antibody panel

\section{CSF}

Acid fast stain

Bacterial, viral, mycobacterial, and fungal cultures

Cryptococcal antigen

Epstein-Barr virus PCR

Gram stain

Herpes simplex virus PCR

La Crosse virus PCR

West Nile virus antibody panel

All the studies were negative.

\section{DISCUSSION}

As many as $40 \%$ of cases of status epilepticus (SE) fail to respond to first- and second-line antiepileptic medications. ${ }^{2}$ These cases are defined as refractory SE (RSE). When RSE develops in a previously healthy individual, and cannot be explained by an obvious underlying cause after 48 hours of initial investigations, the condition is termed new-onset RSE (NORSE). ${ }^{3}$ In the largest case series published to date, half of all cases of NORSE remained cryptogenic

\section{Figure Microscopic findings in left parahippocampal gyrus (hematoxylin \& eosin stain)}
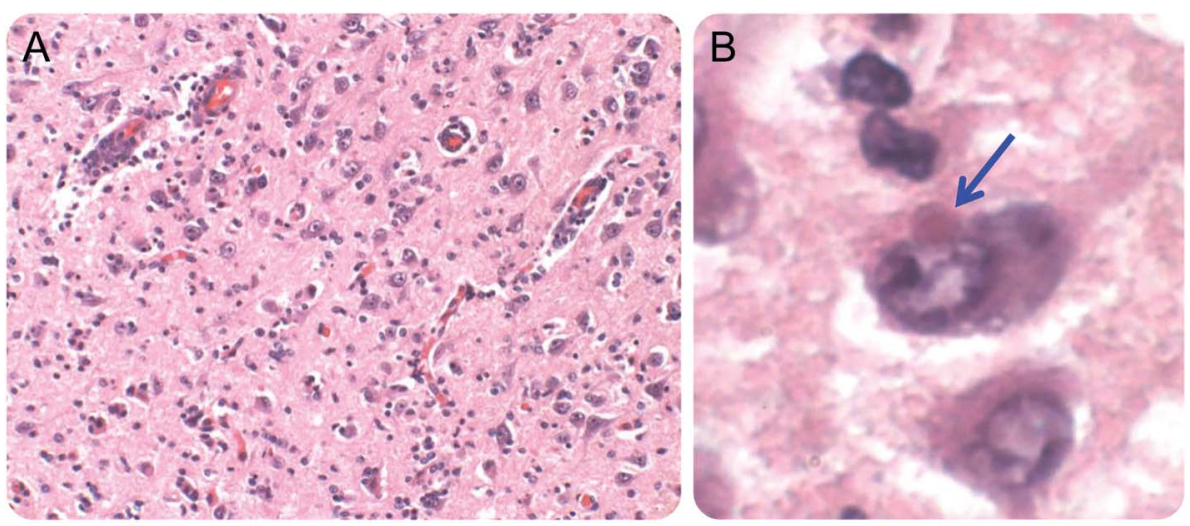

(A) Low-power image demonstrates diffuse intraparenchymal inflammation showing lymphocytic and polymorphonuclear cell infiltration with focal neuronophagia. (B) High-power image reveals sharply defined, homogenously eosinophilic intracytoplasmic inclusion bodies, consistent with Negri bodies (arrow). 
despite extensive investigations. When an underlying etiology was identified, autoimmune and paraneoplastic encephalitides accounted for $70 \%$ of cases, while infectious processes were much less frequent. ${ }^{3}$ NORSE secondary to rabies encephalitis is rare.

Rabies encephalitis is an acute CNS infection caused by the rabies virus $(R A B V)$, an RNA rhabdovirus. There is involvement of the brainstem and cerebrum, with early predilection for the limbic system that accounts for behavioral changes seen early in the disease. Incubation period, although highly variable, is on average 1-2 months. Once clinical onset of symptoms ensues, rabies encephalitis has a fulminant course and is almost uniformly fatal, with an average survival of less than 6 days. ${ }^{4}$ Seizures are rare. ${ }^{5}$ In the United States, in up to $80 \%$ of indigenous cases, there is no clear evidence of exposure to a rabid animal. ${ }^{6}$ Routine CSF analysis cannot differentiate rabies from other viral encephalitides. Elevated protein and absence of robust cellular responses in the CSF have been reported in both encephalitic and paralytic rabies. ${ }^{5}$

Although brain MRI in human rabies encephalitis can show nonspecific abnormalities, T2 hyperintensities of the basal ganglia or thalami are often identified. ${ }^{7}$ MRI findings may be influenced by the multiple metabolic comorbidities seen in these patients. ${ }^{4}$ Normal MRI, like in our patient, appears to be uncommon. Similarly, MRI can be unrevealing in many cases of NORSE, where only $62 \%$ demonstrate any abnormalities on MRI. ${ }^{3}$

In retrospect, as suggested by the presence of myoglobin cast nephropathy at autopsy, we suspect that the development of rhabdomyolysis in our patient might have been explained at least in part by propofol-related infusion syndrome. Coincidentally, our patient was found to have biopsy-proven disseminated histoplasmosis despite negative complement fixation. As there was no personal or family history of immunodeficiency, the co-occurrence of both infections and the patient's residence in a geographic area where numerous caves are found suggested caves as a common source of exposure. Although no viral genetic studies were performed, we speculate that bat $R A B V$ variant could have been the cause of our patient's infection.

\section{REFERENCES}

1. Rupprecht CE, Hanlon CA, Hemachudha T. Rabies re-examined. Lancet Infect Dis 2002;2:327-343.

2. Mayer SA, Claassen J, Lokin J, Mendelsohn F, Dennis LJ, Fitzsimmons BF. Refractory status epilepticus: frequency, risk factors, and impact on outcome. Arch Neurol 2002;59:205-210.

3. Gaspard N, Foreman BP, Alvarez V, et al. New-onset refractory status epilepticus: etiology, clinical features, and outcome. Neurology 2015;85:1604-1613.

4. Hemachudha T, Ugolini G, Wacharapluesadee S, Sungkarat W, Shuangshoti S, Laothamatas J. Human rabies: neuropathogenesis, diagnosis, and management. Lancet Neurol 2013;12:498-513.

5. Hemachudha T, Tirawatnpong S, Phanthumchinda K. Seizures as the initial manifestation of paralytic rabies. J Neurol Neurosurg Psychiatry 1989;52:808-810.

6. Messenger SL, Smith JS, Rupprecht CE. Emerging epidemiology of bat-associated cryptic cases of rabies in humans in the United States. Clin Infect Dis 2002;35:738-747.

7. Awasthi M, Parmar H, Patankar T, Castillo M. Imaging findings in rabies encephalitis. AJNR Am J Neuroradiol 2001;22:677-680.

Received December 19, 2016. Accepted in final form April 17, 2017.

\section{AUTHOR CONTRIBUTIONS}

M.F. Villamar: case concept and design, acquisition of data, interpretation of data, manuscript writing. J. H. Smith: case concept and design, interpretation of data, critical revision of manuscript for intellectual content. D. Wilson: case concept and design, interpretation of data, critical revision of manuscript for intellectual content. V.D. Smith: case concept and design, interpretation of data, critical revision of manuscript for intellectual content.

\section{ACKNOWLEDGMENT}

The authors thank Dr. Gerard J. Nuovo for providing information regarding methodology of the rabies RT-PCR studies performed in this patient. 


\section{STUDY FUNDING}

No targeted funding reported.

\section{DISCLOSURES}

M.F. Villamar: research support, academic entities (1) Alpha Omega Alpha Postgraduate Award, (2) seed grant from EpiC, the University of Kentucky Epilepsy Research Center. J.H. Smith, D. Wilson, and V.D. Smith report no disclosures. Full disclosure form information provided by the authors is available with the full text of this article at Neurology.org/cp.

\section{Neurology ${ }^{\circledR}$ Genetics Call For Papers}

\section{Neurology}

Genetics

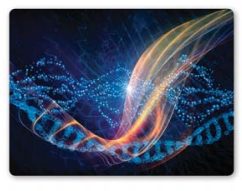

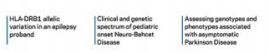

Neurology: Genetics is an open access, online only journal that will provide neurologists with outstanding original contributions that elucidate the role of genetic and epigenetic variation in diseases and biological traits of the central and peripheral nervous system. We welcome all submissions. For more information on how to submit, visit http://www.neurology.org/site/ gen/gen2.xhtml. 